\title{
ENSEÑANDO PALABRAS MEDIANTE LIBROS ILUSTRADOS: El APRENDIZAJE TEMPRANO DE SUSTANTIVOS Y ADJETIVOS*
}

\section{TEACHING WORDS THROUGH PICTURE-BOOKS: EARLY LEARNING OF NOUNS AND ADJECTIVES}

\author{
Florencia Mareovich ${ }^{* *}$, Andrea S. Taverna*** y Olga A. Peralta ${ }^{* * *}$
}

\begin{abstract}
"Este trabajo forma parte de la Tesis Doctoral de la primera autora. Directora de tesis: Dra. Olga A. Peralta. Trabajo financiado con subsidios de la Agencia Nacional de Promoción Científica y Tecnológica (ANPCyT), del Consejo Nacional de Investigaciones Científicas y Técnicas (CONICET) y de la Secretaría de Investigaciones de la Universidad Abierta Interamericana (UAI) otorgados a la tercera autora, y por becas doctorales de ANPCyT y CONICET otorgadas a la primera autora.

"Psicóloga y Doctora en Psicología. Becaria Posdoctoral del Consejo Nacional de Investigaciones Científicas y Técnicas (CONICET) y Profesora Adjunta de la asignatura Psicología Educacional en la Universidad Abierta Interamericana (UAI) Sede Rosario. E-Mail: mareovich@irice-conicet.gov.ar

***Psicóloga, Profesora y Doctora en Psicología. Miembro de la Carrera del Investigador Científico del Consejo Nacional de Investigaciones Científicas y Técnicas (CONICET) y Profesora Titular de la asignatura Seminario de Tesina en el Instituto Universitario del Gran Rosario. E-Mail: ataverna@conicet.gov.ar ****Licenciada en Psicología, Master in Science y Doctora en Psicología. Miembro de la Carrera del Investigador Científico del Consejo Nacional de Investigaciones Científicas y Técnicas (CONICET) y Profesora Titular de la asignatura Psicología Educacional en la Universidad Abierta Interamericana (UAI) Sede Rosario. Instituto Rosario de Investigaciones en Ciencias de la Educación (IRICE - CONICET). Boulevard 27 de Febrero 210 bis. (2000) Rosario, República Argentina. E-Mail: peralta@irice-conicet.gov.ar Las autoras agradecen la colaboración recibida para la realización del estudio a los niños e instituciones participantes.
\end{abstract}

\section{RESUMEN}

El objetivo del trabajo que se informa fue investigar el aprendizaje de adjetivos y sustantivos mediante libros ilustrados en niños de tres años de edad. Se diseñaron dos estudios con una metodología cuasi-experimental en los que se enseñó a los niños un sustantivo o un adjetivo. Luego los participantes debían extender la palabra aprendida primero al objeto representado por la imagen, y luego a un nuevo ejemplar de la misma categoría de objeto (sustantivo) o propiedad de objeto (adjetivo). El Estudio 1 comparó el aprendizaje de sustantivos y adjetivos. Se encontró que los niños aprendieron el sustantivo pero no el adjetivo, lo que indicaría que para ellos aprender adjetivos a través de libros con imágenes es más difícil que aprender sustantivos.
El Estudio 2 exploró el impacto de la información descriptiva sobre el aprendizaje de un adjetivo que refería a una propiedad visual (estampado). Esta información destacaba, por un lado, el núcleo del concepto, el estampado, y por otro la intención comunicativa del interlocutor. Se contrastó la ejecución de los niños en función de la información provista por la experimentadora. Los datos obtenidos muestran que si los niños reciben información descriptiva sobre el aspecto de la imagen que debe conectarse con la palabra, logran relacionarlo con la propiedad a la que refiere, aprendiendo el adjetivo. Los resultados de esta investigación aportan información relevante acerca de la relación entre el aprendizaje del léxico y el desarrollo conceptual y subrayan la relevancia del contexto sociopragmático en el aprendizaje de palabras. 
Palabras clave: Aprendizaje del léxico; Sustantivos; Adjetivos; Información descriptiva; Imágenes.

\section{ABSTRACT}

Picture-books reading are a very common practice between children and adults. In these interactions parents assume that their children learn lots of things, especially words. But, is this interaction effective as a word learning device? Is it the same to teach different kind of words? The main question of this research arises in the intersection of two fields: symbolic comprehension of pictures and word learning. Three main ideas guided this research. Symbolic understanding of pictures is a very complex process that involves cognitive and social skills. The sociopragmatic context has an impact in the cognitive processes involved in word learning. There is a strong relationship between word learning and conceptual development. The aim of this research was to explore 3-year-old children's learning of nouns and adjectives in a picture-book reading situation. We designed two studies using a cuasiexperimental approach. In Study 1 we compared two groups of children: one learned a noun (category condition) and the other an adjective (property condition). The noun was pompe, a word that does not exist but has a Spanish morphologic structure. The adjective, pompeado, was built based on the word pompe, adding the suffix-ado; suffix marks that the word is an adjective. Toddlers were exposed to the new words during a picturebook reading interaction with the experimenter, then, they had to solve two successive tests. In the first test of the category condition participants had to apply the new noun learned via pictures to the real object. In the second test they had to extend the new noun to a new exemplar, an object with the same shape but different color. In the first test of the property condition, participants had to apply the new adjective learned via pictures to the real object with the property. In the second test they had to extend the adjective to a new exemplar, a new object with the same target-property. The second tests were taken as strong indicators of word learning as they involved some kind of generalization. In the first test both groups, category and property, applied the word to the real object. In the second test, while children in the category condition extended the noun to another exemplar, children in the property condition did not extend the adjective to another exemplar with the same property. In a second study we explored if children need more cues to extend the adjective to another exemplar with the same property, and truly learn the adjective. Study 2 investigated the impact of descriptive information in word learning. We set two groups on the basis of the information provided by the experimenter. One group received descriptive information highlighting the specific aspect of the picture that had to be mapped with the property. The other group did not receive this additional information. We found that only the participants that received descriptive information extended the word pompeado to a new exemplar with the target property. We propose that the descriptive information worked highlighting the communicative intention of the experimenter as well as the core of the concept. Underscoring the perceptual feature allowed much more than making a superficial association, as the descriptive information pointed directly to the heart of the concept. Overall, the results of this research are consistent with previous studies that found that it is more difficult for young children to learn adjectives than nouns. The results also highlight the strong relationship between word learning and conceptual development. This research provides strong evidence concerning the importance of socio the pragmatic context on referential understanding and word learning.

Key words: Lexical learning; Nouns; Adjectives; Descriptive information; Pictures.

\section{INTRODUCCIÓN}

Los libros ilustrados son medios simbólicos a través de los cuales los adultos suelen enseñar palabras a los niños. La lectura conjunta de libros ilustrados por parte de padres 
y niños pequeños es muy frecuente, al menos en algunas culturas y niveles socioeconómicos (DeBaryshe, 1993; Huebner, 2000; Lonigan, 1994; Payne, Whitehurst \& Angell, 1994). Numerosas investigaciones han indagado las características y dinámica de estas interacciones (DeLoache \& Peralta, 1987; Ninio \& Bruner, 1978; Peralta, 1995; Snow \& Goldfield, 1983). No obstante, la pregunta acerca de si los niños efectivamente aprenden en estos intercambios ha sido poco estudiada.

La investigación que se informa estudió el aprendizaje de palabras, específicamente de sustantivos y adjetivos, por parte de niños pequeños en un contexto de interacción con libros ilustrados. En este trabajo confluyen dos campos de investigación: el desarrollo de la comprensión de imágenes y el aprendizaje del léxico.

\section{DESARROLLO DE LA COMPRENSIÓN DE IMÁGENES}

Para aprender a través de imágenes es crucial comprender su rol referencial. Esto implica entender que las imágenes vienen a mostrar algo acerca del mundo. Desde muy temprano los niños dan indicios de reconocer objetos y personas presentes en imágenes y distinguir entre ellas y sus contrapartidas reales (Barrera \& Maurer, 1981; DeLoache, Strauss \& Maynard, 1979; Rose, 1977).

A pesar de estas tempranas capacidades perceptuales, los niños tienen dificultades en comprender la relación simbólica imagen referente. Tanto padres como investigadores han informado que los niños a menudo confunden una foto con su referente tratando de tomar los objetos representados en fotografías (Murphy, 1978; Perner, 1991; Wener \& Kaplan, 1963). Estudios experimentales también evidenciaron este fenómeno (DeLoache, Pierroutsakos, Uttal, Rosengren \& Gottlieb 1998; Pierroutsakos \& DeLoache, 2003).

La comprensión de imágenes es el resultado de un proceso cognitivo complejo donde reconocimiento no debe confundirse con comprensión (Sigel, 1978). Mientras que el primero sólo requiere habilidades perceptuales, según algunos autores, innatas o con muy poca necesidad de aprendizaje, la segunda requiere un entramado de habilidades cognitivas, sociales y perceptuales. Comprender el rol representacional de imágenes implica la decodificación de la información presente en ellas y su correcta vinculación con lo que representan (Ittelson, 1996). Además los niños deben desplegar habilidades cognitivas específicas como por ejemplo, el mecanismo que DeLoache (1987) denominó doble representación ya que se deben mantener mentalmente dos representaciones, la del objeto concreto y la de la entidad que representa.

Numerosas investigaciones han encontrado que los niños comprenden la relación que une una imagen con su referente entre los 18 y 30 meses de edad (Ganea, Allen, Butler, Carey \& DeLoache, 2009; Harris, Kavanaugh \& Dawson, 1997; Peralta \& Salsa, 2011; Preissler \& Carey, 2004). Sin embargo, a estas edades tempranas la comprensión de imágenes se ve afectada por múltiples variables entre las que se destacan la similitud perceptual entre el símbolo y el referente y la instrucción que el adulto provee al niño (De Loache, Peralta \& Anderson, 1999).

Además la comprensión simbólica de imágenes dependerá del uso que se haga de ellas. Una imagen puede usarse como una representación específica, referida a un evento o entidad en particular, o como una representación genérica, denotando un tipo o categoría de objeto o evento. Las imágenes que aparecen en libros ilustrados suelen utilizarse como representaciones genéricas (DeLoache \& Burns, 1994).

En resumen, si bien los bebés y niños muy pequeños poseen habilidades para percibir imágenes y reconocer objetos representados, comprender su estatuto simbólico y utilizarlas en diferentes contextos requiere habilidades específicas. Por otra parte, el desarrollo de la comprensión de imágenes es posible gracias a la interacción del niño con su medio social, lo que le permitirá comprender las intenciones comunicativas de los demás, y utilizar a las imágenes como símbolos (Bloom \& Markson, 1998; Callaghan, 2008). 


\section{ApRENDIZAJE DEL LÉXICO}

En el campo de la Filosofía, la Psicología y la Psicolingüística se han propuesto diferentes aproximaciones al estudio de la adquisición del lenguaje, las que presentan marcadas divergencias pero también algunos puntos de encuentro (para una revisión ver Taverna \& Peralta, 2009). En cuanto al aprendizaje de palabras, en la actualidad existen dos enfoques claramente contrapuestos (Geraghty, Waxman \& Gelman, 2014):

Por un lado, los asociacionistas que entienden que los niños inicialmente en el desarrollo los significados de palabras incorporan solo contenidos perceptivos y sensoriales agrupados en asociaciones, sólo más tarde incorporarán el componente conceptual a esos primeros significados (Sandhofer \& Smith, 2004; Sloutsky, 2010; Smith \& Samuelson, 2006). Por otro lado, varios exponentes provenientes de diferentes líneas de trabajo confluyen en la propuesta de una perspectiva que integra el desarrollo conceptual al estudio del desarrollo del lenguaje en general y del aprendizaje de palabras y la formación del significado, en particular. Esta aproximación defiende la idea que los primeros significados de palabras incorporan desde el inicio contenido conceptual y contenido perceptivo, y que en el proceso de establecer el significado de una palabra, los infantes interpretan ese contenido en el contexto de sus hipótesis y expectativas sobre palabras y conceptos (Booth, Waxman \& Huang, 2005; Carey, 2009; Gelman \& Waxman, 2009; Putnam, 1973; Spelke, 2000).

También asumen que ni la capacidad de identificar una nueva palabra ni la capacidad de representar conceptos garantizan que el niño asociará conjuntamente con éxito estas unidades lingüísticas y conceptuales. Por el contrario, este enfoque convalida las hipótesis centrales de las perspectivas funcionalistas o pragmáticas que afirman que para asignar correctamente palabras a sus referentes, el niño debe inferir información acerca de los objetivos e intenciones de los hablantes de su entorno (ver Woodward \& Markman, 1998, para una excelente visión general, también Baldwin \& Moses, 2001; Meltzoff, 2002; Tomasello \& Olguín, 1993; Woodward, 2004).

Existe evidencia que apoyaría la perspectiva que integra el desarrollo conceptual al desarrollo del lenguaje. Hacia los 12 meses de edad los infantes extienden nuevos sustantivos a categorías de objeto en base a principios específicos y no a agrupamientos basados en la similitud (Booth et al., 2005; Waxman, 1999), y que los utilizan para realizar inferencias acerca de nuevos objetos (Graham, Booth \& Waxman, 2012; Keates \& Graham, 2008; Xu, Cote \& Baker, 2005). Resultados como estos indican que los infantes aprecian que una palabra es un símbolo cuyo ámbito referencial se extiende más allá del objeto en particular con el que se ha emparejado.

Los estudios en esta línea también muestran que la adquisición de esta habilidad ocurre gradualmente, primero estableciéndose asociaciones muy generales entre palabras y conceptos y más tarde, emergiendo asociaciones específicas entre diferentes clases de palabras o formas gramaticales (verbos, adjetivos) y diferentes clases de conceptos (categorías de evento y estado, propiedades de objeto). Se sugiere que los infantes descubren que hay formas gramaticales distintas cuando empiezan a notar las distintas formas léxicas o marcos gramaticales en las que se producen distintos tipos de palabras (Brent \& Cartwright, 1996; Mintz, 2003; Mintz, Newport \& Bever, 2002).

En este sentido, algunos estudios han mostrado que los infantes primero identifican los sustantivos y los asocian específicamente a categorías de objeto. La evidencia de registros observacionales, reportes parentales y estudios experimentales indica que el privilegio de los sustantivos en el desarrollo del vocabulario infantil ha sido confirmado en un gran número de lenguas, incluso en aquellas que por sus características estructurales podrían privilegiar a otras formas gramaticales como los verbos (cf., Gleitman, Cassidy, Nappa, Papafragou \& Trueswell, 2005; Golinkoff \& Hirsh-Pasek, 2008; Pye, 1992; Tardif, Fletcher, Liang, Zhang, Kaciroti \& Marchman, 2008). 
Las asociaciones entre palabras y conceptos posteriores a los sustantivos (por ejemplo, adjetivos y verbos) aparecerían sobre la asociación sustantivo-categoría de objeto en función de la experiencia con determinadas categorías gramaticales y sus significados asociados en la lengua que se está adquiriendo (Waxman \& Lidz, 2006).

Atendiendo a los adjetivos, se debe tener en cuenta que están muy presentes en el discurso que los adultos dirigen a los bebés y niños. Si bien los pequeños pueden detectar muchas propiedades que son denotadas por adjetivos (por ejemplo, texturas o colores) no las consideran como candidatas para el significado de las palabras. Cuando se les presenta un nuevo adjetivo (rojo) en el contexto de un objeto novedoso (auto rojo), los pequeños tienden a interpretar ese adjetivo como el nombre del objeto en lugar de su propiedad. Este fenómeno ha sido documentado en niños de hasta tres años (Hall \& Lavin, 2004; Markman \& Jaswal, 2004).

Las características del sustantivo al cual el adjetivo modifica ha mostrado tener gran impacto en la posibilidad de extenderlo a otro ejemplar. Waxman y Markow (1998) encontraron que es más sencillo para los niños extender un adjetivo a otro ejemplar que comparta la propiedad si ambos pertenecen a la misma categoría básica (por ejemplo, todos serpientes). El impacto de las categorías de nivel básico es un sesgo que se ha observado hasta los tres años de edad (Hall \& Lavin, 2004; Klibanoff \& Waxman, 2000). Además, se ha comprobado que la interpretación que los niños hacen de un adjetivo nuevo varía en función de la familiaridad del sustantivo que modifica (Hall \& Waxman, 1993; Hall, Waxman \& Hurwitz, 1993).

Por otra parte, Hall, Williams y Be'Langer (2010) exploraron el impacto de pistas tanto socio-pragmáticas como léxicas en el aprendizaje de un adjetivo. Las pistas socio-pragmáticas serían acciones que ponen de relieve el referente de la palabra (como indicar la propiedad). Las formas léxicas aludirían a la ubicación donde se presentaba la palabra en la frase. Los autores encontraron que a los tres años de edad los niños utilizaban pistas léxicas, pero no pistas socio-pragmáticas para aprender un adjetivo.

En suma, las investigaciones en aprendizaje del léxico confirman que: (a) los infantes utilizan palabras en tanto son entidades simbólicas y no meras asociaciones perceptivas, (b) el proceso de adquisición de los sustantivos se adquiere antes que el resto de las formas gramaticales (por ejemplo, que los adjetivos) en tanto constituyen la base fundante de la adquisición léxica posterior y (c) para adquirir cualquiera de las formas gramaticales los niños se valen de diferentes tipos de pistas: léxico-sintácticas y conceptual-referenciales, que se actualizan en función de las hipótesis y expectativas que los niños poseen sobre palabras y conceptos, $y$ también de pistas pragmático-intencionales.

\section{APRENDIENDO PALABRAS MEDIANTE IMÁGENES}

En la conjunción de las dos líneas de investigación mencionadas, la comprensión de imágenes y el aprendizaje de palabras, se encuentran los trabajos que exploran la enseñanza de palabras a través de imágenes impresas. Estudios recientes realizados con niños hablantes del idioma inglés encontraron que los niños pequeños pueden aprender sustantivos a través de imágenes (Ganea, Bloom Pickard \& DeLoache, 2008; Ganea et al., 2009; Geraghty et al., 2014; Preissler \& Carey, 2004; Tare, Chiong, Ganea \& De Loache, 2010). Sin embargo, el aprendizaje de adjetivos ha sido poco estudiado (Mareovich, 2013).

Para el estudio que se informa se diseñaron dos estudios que consistían en enseñar palabras nuevas, sustantivos o adjetivos, en el marco de una interacción con libros ilustrados. Las nuevas palabras fueron presentadas en los marcos gramaticales correspondientes al español. En este sentido, ya se le otorgaba a los niños una pista léxica o sintáctica. La tarea consistía en una interacción conjunta por lo que las palabras se presentaban en sus contextos de uso.

Los objetivos de esta investigación fueron los siguientes: (1) comparar el aprendizaje de 
un sustantivo con el de un adjetivo que refería a una propiedad visual (estampado), en un contexto de interacción con libros ilustrados y (2) investigar si los niños pueden asociar la palabra nueva, presentada como adjetivo, a una propiedad visual si además de las pistas sintácticas-léxicas reciben una pista conceptual-referencial y se explicitan las pistas socio-pragmáticas.

En relación al primer objetivo se hipotetizó que si a los niños pequeños se les presentan palabras nuevas, tanto en forma de sustantivos como adjetivos en sus correspondientes marcos gramaticales, los niños considerarán tales palabras como candidatos para categorías de objeto, por lo que aprenderán solo sustantivos y no adjetivos.

La hipótesis vinculada al segundo objetivo era que si se describe en qué consiste la propiedad al señalar y explicitar el aspecto de la imagen que debe relacionarse con el adjetivo, los niños pueden asociar la palabra con su referente.

\section{MÉTOdO}

Se llevaron a cabo dos estudios. Para ambos se diseñaron tareas de elección forzada y palabra extendida (Gentner \& Namy, 1999; Smiley \& Brown, 1979). Se consideró que el aprendizaje de una palabra tiene lugar si el niño, por un lado, extiende la palabra (sustantivo o adjetivo) al objeto concreto representado por la imagen, y por otro lado si generaliza esta palabra a otro ejemplar de la misma categoría, sea ésta una categoría de objeto (para sustantivos) o una propiedad de objeto (para adjetivos).

El primer estudio comparó el aprendizaje de un sustantivo con el de un adjetivo que refería a una propiedad visual (estampado), en un contexto de interacción con libros ilustrados.

El segundo estudio exploró el aprendizaje de un adjetivo en niños pequeños, comparando este aprendizaje con información descriptiva (con información de contenido conceptual-referencial y socio-pragmática) y sin información descriptiva (solo se presentaba la palabra en su contexto léxico-gramatical).

\section{PARTICIPANTES}

Participaron 68 niños (34 niñas y 34 niños) de 36 meses (rango de edad: 35 a 37 meses) asignados al azar a cuatro grupos: Categoría - Propiedad (Estudio 1) y Sin descripción - Con descripción (Estudio 2). Todos los participantes eran de nivel sociocultural medio y fueron contactados a través de los jardines de infantes a los que acudían en la zona céntrica de la ciudad de Rosario (Provincia de Santa Fe, Argentina). El nivel de escolaridad de los padres y las madres era terciario o universitario, completo o incompleto. Los padres y las madres trabajaban en sus profesiones o en actividades comerciales y algunas madres eran amas de casa. Según los registros de los docentes y de las instituciones los niños presentaban un desarrollo cognitivo esperado para su edad y no presentaban patologías lingüísticas. Se obtuvo un consentimiento informado por escrito tanto de los padres como de las instituciones.

\section{MATERIALES}

Imágenes: Dos libros de cartón con 12 fotografías de $11 \times 11 \mathrm{~cm}$ cada uno (ver Anexo, Figura 1).

En la condición Categoría (Estudio 1) se presentaron imágenes de dos objetos desconocidos, cada uno aparecía en cuatro oportunidades. La imagen de uno de ellos, la del objeto-meta fue relacionada con la palabra nueva: pompe. La otra imagen funcionó como distractor. Además, se emplearon imágenes de objetos familiares (auto, pelota, manzana, anteojos, flor y perro de peluche).

En las condiciones Propiedad (Estudio 1) y Sin descripción y Con descripción (Estudio 2) se presentaron seis imágenes de objetos con propiedades convencionales (pato amarillo, oso peludo, arcoíris colorido, papel arrugado, pelota redonda y cielo nublado) y dos 
de objetos con propiedades no convencionales (caballo a lunares y tren a cuadros). Una propiedad (lunares) funcionó como propiedad-meta para la mitad de los niños (Orden 1). La otra propiedad (cuadros) funcionó como propiedad-meta para la otra mitad de los niños (Orden 2).

Objetos: En la condición Categoría (Estudio 1) se utilizaron tres objetos: el meta, el distractor y un nuevo ejemplar del objeto meta (misma forma pero diferente color). En la condición Propiedad (Estudio 1): Sin descripción y Con descripción (Estudio 2) se utilizaron cuatro objetos: caballo a lunares, tren a cuadros, flor a lunares (Orden 1) y vestido a cuadros (Orden 2).

\section{Procedimiento}

Primero, la experimentadora mostrando un títere le decía al niño: "A Jack le encanta tomar fotos de sus juguetes y tiene un libro lleno de fotos, ¿lo vemos?"

\section{1.- Fase de enseñanza}

En la condición Categoría (Estudio 1) se enseñó un sustantivo nuevo (pompe). La experimentadora mostraba el libro al niño: a los objetos familiares los nombraba una vez (Mira la pelota), mientras que al objeto-meta lo nombraba tres veces (Mira el pompe, este juguete lo inventó Jack, ¿viste el pompe?, aquí está el pompe), al objeto distractor lo señalaba sin nombrarlo (Mira esto, es otro juguete que Jack inventó).

En la condición Propiedad (Estudio 1) y Sin descripción (Estudio 2) se enseñó un adjetivo nuevo (pompeado). El objeto con la propiedad-meta aparecía cuatro veces, y la experimentadora lo nombraba tres veces en cada oportunidad (Este caballo / tren es pompeado; mira, Jack dice que esto es pompeado; ¿viste que lindo este caballo/tren pompeado?). La experimentadora se dirigía al objeto con la propiedad distractora pero sin nombrarlo (Mira esto, iqué lindo! viste?). Los objetos con propiedades convencionales fueron nombrados una vez (;Mira! un arcoíris colorido).
En la condición Con descripción (Estudio 2) los participantes aprendieron la palabra pompeado recibiendo información descriptiva acerca de las características de la imagen, un estampado, con la cual el niño debía relacionar la palabra. En la fase de entrenamiento la experimentadora nombraba a los objetos con propiedades convencionales describiendo la propiedad (por ejemplo: Mira, este arcoíris, viste todos estos colores, es colorido). A la propiedad-meta la nombraba tres veces acompañada de la descripción de la propiedad y señalamiento de los detalles del estampado (por ejemplo: Mira esto es pompeado, Jack dice que esto es pompeado porque tiene todos estos dibujitos ¿viste qué lindo es esto pompeado?). La experimentadora se refería al objeto que poseía la propiedad que funcionaba como distractor, describiéndola pero sin nombrarla (por ejemplo: Mira esto, ¿lo ves? ¿Viste estos dibujitos que tiene?).

\section{2.- Fase de familiarización}

En esta fase se intentó familiarizar al niño con el tipo de preguntas que serían formuladas en la fase de prueba. La experimentadora mostraba dos imágenes de objetos conocidos o de objetos que presentaban propiedades convencionales (utilizados en la fase de entrenamiento), según condición, y se le pedía que señalara uno de ellos (por ejemplo: ¿Cuál de estos es un oso?; ¿Dónde hay algo amarillo?).

3.- Fase de presentación de los objetos

La experimentadora mostraba a los niños los dos objetos nuevos o los objetos que contenían propiedades no convencionales, según condición, para evitar que la elección del objeto en la fase de prueba fuera simplemente por la novedad.

\section{4.- Fase de prueba}

En esta fase los niños debían realizar dos elecciones ante una pregunta de la experimentadora: ¿Cuál de estos es un pompe? (condición Categoría) o ¿Cuál de estos es pompeado? (condición Propiedad, Sin descripción y Con descripción). En estas tareas se evalúa la extensión de la palabra enseñada 
primero a un objeto concreto, representado por la fotografía, y luego a otro ejemplar de la misma categoría.

Comprensión simbólica: Elección entre el objeto-meta o el objeto con la propiedad-meta (según condición) y el objeto distractor. En este caso se explora la extensión por parte del niño del sustantivo o adjetivo aprendido a través de una imagen a objetos reales. Esta extensión estaría indicando que el niño comprende que esa imagen refiere a ese objeto real.

Generalización: Elección entre un nuevo objeto-meta, misma forma pero diferente color, o de un nuevo objeto con la propiedad meta (según condición) y el objeto distractor (Ver Anexo, Figura 2). Se exploró la extensión de la palabra enseñada a otro ejemplar de la misma categoría de objeto o a otro objeto que posea la propiedad.

\section{ANÁLISIS DE DATOS}

Los datos registrados en las interacciones fueron volcados a un protocolo para su análisis. Se tomó en cuenta la primera elección del niño. El participante debía señalar o explicitar verbalmente su elección dirigiéndose a la experimentadora. Las variables dependientes, constituidas por las elecciones de los niños en las tareas se estructuraron como variables nominales dicotómicas (correcto-incorrecto).

El primer análisis consistió en contrastar las respuestas de los participantes contra el azar utilizando la Prueba de ji-cuadrado Bondad de Ajuste. Luego, se comparó la ejecución de los grupos por condición experimental mediante la prueba exacta de Fisher. Para llevar a cabo los análisis se utilizó el programa estadístico SPSS ${ }^{\circledR}$ versión 20 .

\section{ESTUDIO 1}

El propósito de este estudio fue explorar el aprendizaje de sustantivos y adjetivos en un contexto de interacción con libros ilustrados con fotografías. Se contrastó la ejecución de niños de tres años de edad en el aprendizaje de palabras que denotan dos tipos de referentes: Categoría de objeto y Propiedad de objeto.

El sustantivo escogido fue una seudopalabra (pompe) extraída del Test de Lectura y Escritura en Español LEE (Defior Citoler et al., 2006). Se trata de una palabra desconocida e inexistente que no figura en el diccionario de la Real Academia Española (RAE, 2012), pero cuya estructura fonológica se asemeja a algunas de las palabras de nuestra lengua, de tal manera de no resultar extraña ni interferir en el aprendizaje. Se construyó el adjetivo, pompeado, tomando como base la palabra pompe, y agregándole un sufijo común para adjetivos de la lengua española, ado.

\section{RESULTADOS Y DISCUSIÓN}

\section{COMPRENSIÓN SIMBÓLICA}

La ejecución superó los niveles del azar en ambos grupos: Categoría $\left[\chi^{2}(1\right.$, $N=17)=13.23 ; p<.01]$ y Propiedad $\left[\chi^{2}(1\right.$, $N=17)=13.23, p<.01]($ ver Gráfico 1$)$. En Categoría, 16 niños (94\%) aplicaron la palabra pompe al objeto real, o sea, establecieron conexiones entre la imagen y el objeto. En la condición Propiedad, 16 niños $(94 \%)$ extendieron la palabra pompeado al objeto que poseía la propiedad. Esta elección indicaría que en ambos casos los niños comprendieron simbólicamente la imagen, es decir, comprendieron que esa imagen refería al objeto real presentado.

Sin embargo, en el caso del aprendizaje del adjetivo este patrón de elección podría denotar que los niños realizaron una conexión entre la totalidad de la imagen y del objeto. Es decir, la elección del objeto pompeado podría no indicar la detección del referente específico: la propiedad. Así la palabra pompeado sería considerada como un sustantivo y se relacionaría con una categoría de objeto. Algunas verbalizaciones espontáneas de los participantes parecen apoyar la 
interpretación en esa dirección. Algunos niños repetían: esto es un pompeado. La sintaxis de la frase con la presencia de la palabra un, denota que pompeado para ellos podría ser considerado un objeto.

\section{GENERALIZACIÓN}

En la condición Categoría, 16 niños (94\%) escogieron el nuevo ejemplar del objeto meta y uno $(6 \%)$ el objeto distractor $\left[\chi^{2}(1, N=17)=13.23 ; p<.01\right]$. Por el contrario, en la condición Propiedad, tres niños $(18 \%)$ señalaron el nuevo ejemplar con la propiedad-meta y $14(82 \%)$, el objeto con la propiedad distractora, elección también superior a los niveles del azar $\left[\chi^{2}(1\right.$, $N=17)=7.11 ; p<.01]$ (ver Gráfico 1$)$.

También se comparó la ejecución de los niños en la prueba de Generalización por condición experimental: Categoría vs Propiedad encontrándose diferencias significativas entre los grupos (prueba exacta de Fisher, $p<.01)$. El coeficiente Phi $(-.770, p<.01)$ indica que la relación es fuerte.

Los datos reflejan que los participantes de la condición Categoría conectaron la palabra con el referente, categoría de objeto, mientras que los niños de la condición Propiedad no mostraron respuestas referenciales, es decir no conectaron el adjetivo con la propiedad. Durante la interacción un participante de la condición Propiedad, sentenció señalando el nuevo ejemplar pompeado: ;Este no! ;Es una flor! Esto indicaría que para el niño el objeto pertenecía a una categoría de objeto conceptual de nivel básico: flor, y por lo tanto no podía pertenecer a otra categoría: pompeado. Es decir que el pertenecer a la categoría de objeto flor imposibilitaría incluir al ejemplar flor en otra categoría conceptual (pompeado), lo que además estaría mostrando una limitación en la flexibilidad a la hora de categorizar según dos dimensiones a un mismo objeto.

Los resultados aquí presentados, que ilustran el aprendizaje de palabras a través de libros con imágenes, son consecuentes con el patrón evolutivo que señala que los niños ad- quieren sustantivos antes que adjetivos. Sin embargo, si se observa a niños de esta edad, es evidente que ya han aprendido, aprenden y utilizan diariamente, una gran cantidad de adjetivos aplicados a múltiples objetos. ¿Requerirán información más específica acerca de la relación entre el adjetivo y su referente para aprender esta nueva palabra? Para responder este interrogante se diseñó el Estudio 2.

\section{ESTUDIO 2}

El Estudio 2 exploró si los niños conectan un adjetivo con una propiedad de objeto y lo aplican a otros ejemplares, si se les proveen pistas más precisas que apunten a explicitar la relación que une a la palabra con la propiedad utilizando acciones de señalamiento del estampado y descripción verbal del mismo. Esta descripción por un lado evidenciaría el rasgo perceptual de la imagen que debe conectarse con el referente (pista conceptual-referencial) y por otro, pondría de manifiesto la intención comunicativa de la experimentadora (pista socio-pragmática). Se establecieron dos condiciones: Sin descripción y Con descripción.

\section{RESULTADOS Y DISCUSIÓN}

\section{COMPRENSIÓN SIMBÓLICA}

La ejecución de ambos grupos superaron los niveles del azar. En el grupo Sin descripción, 16 niños (94\%) extendieron la palabra pompeado al objeto real $\left[\chi^{2}(1, N=17)=13.23\right.$; $p<.01]$. La mayoría de los participantes (13, $76 \%$ ) de la condición Con descripción también optó por el objeto pompeado sobre el objeto con la propiedad distractora $\left[\chi^{2}(1, N=17)=4.76\right.$; $p<.05]$. Esto indicaría que comprendieron la relación entre la imagen y el objeto al cual refiere. Sin embargo, los participantes pudieron haber conectado el adjetivo con la totalidad del objeto (por ejemplo el caballo) y no con la propiedad específica a la cual refería (lunares). 


\section{GENERALIZACIÓN}

En el grupo Sin descripción, 14 niños $(82 \%)$ escogieron el objeto con la propiedad distractora, elección superior al azar $\left[\chi^{2}(1\right.$, $N=17)=7.11 ; p<.01]$; solo tres $(18 \%)$ eligieron el nuevo ejemplar con la propiedadmeta. Los niños de la condición Sin descripción no generalizaron el adjetivo a otro ejemplar que presentaba la misma propiedad; esto indicaría que relacionaron la palabra pompeado con una categoría de objeto, por ejemplo el caballo, y no con la propiedad específica, el estampado.

Por el contrario, en el grupo Con descripción 15 niños (88\%) eligieron el nuevo ejemplar del objeto meta, elección también superior al azar $\left[\chi^{2}(1, N=17)=9.94 ; p<.01\right]$. La información descriptiva otorgada pareció ser decisiva. Por ejemplo, un niño manifestó en presencia del objeto con la propiedad distractora: Este no porque tiene otro dibujito. Estos dichos parecen indicar que los niños reconocieron que la palabra refería a una propiedad de objeto, un estampado, y que dicha propiedad estaba presente en varios objetos diferentes, los cuales pueden ser agrupados en base a esa característica que comparten.

Se encontraron diferencias significativas comparando las condiciones Con y Sin descripción en la prueba de extensión utilizando la prueba exacta de Fisher $(p<.01)$, el coeficiente $p h i$ encontrado $(-.765, p<.01)$ indica que la fuerza de esta relación es fuerte (ver Tabla 1). La instrucción del adulto fue entonces crucial para que los niños detectasen la conexión entre un adjetivo, un aspecto de la imagen y la propiedad a la que refería.

Cabe destacar que los niños en la condición Con descripción aplicaron la palabra no sólo a las categorías de objeto de nivel básico, sino también de nivel supraordenado. Los niños transfirieron la palabra pompeado en el Orden 1 de un animal a una planta, y en el Orden 2 de un medio de transporte a una prenda de vestir. parecería que la pista utilizada, descripción de la propiedad, contribuyó a detectar las similitudes entre dos entidades muy diferentes que compartían una única característica, ese estampado.

\section{DISCUSIÓN GENERAL}

Diariamente los niños aprenden palabras en el seno de interacciones sociales. La mayoría de estos aprendizajes se desarrollan en 'lecciones espontáneas', en las que los hablantes más experimentados, como adultos o niños mayores, ponen en uso su lengua y los aprendices captan los significados de las palabras. Sin embargo, en ocasiones, los padres enseñan deliberadamente vocabulario a sus niños pequeños, siendo los libros ilustrados un medio privilegiado para este fin. ¿Qué es lo que aprenden los niños en estas interacciones?, ¿Qué tipo de input es más efectivo para enseñar un tipo particular de palabra?

Desde el punto de vista de la comprensión de imágenes, en consonancia con investigaciones previas (DeLoache \& Burns, 1994; Ganea et al., 2009; Peralta \& Salsa, 2011), los resultados nos señalan que los niños a los tres años de edad comprenden el rol referencial de estos medios simbólicos. Es decir, tanto aprendiendo sustantivos como adjetivos conectaron la imagen con los objetos a los cuales referían. Los participantes decodificaron la información presente en la imagen y la conectaron con los objetos de la realidad (Ittelson, 1996).

Sin embargo, podríamos preguntarnos qué tipo de representaciones son estas imágenes, ¿son representaciones genéricas o específicas? Cuando se despliega este tipo de interacciones con libros ilustrados usualmente el objetivo es que los niños conozcan las características, por ejemplo, de los perros en general, no de un perro en particular. Por lo tanto las imágenes utilizadas en esta investigación serían representaciones genéricas.

Cuando los participantes debían aprender un sustantivo, el referente, tanto de la imagen cómo la palabra, sería una categoría de objeto, POMPES. Por lo tanto, la extensión de la palabra pompe hacia el mismo objeto observado en la fotografía no solo reflejaría una comprensión referencial de la imagen, sino también la de otros objetos de la misma categoría de objeto, siendo la generalización una tarea fundamental. 
Este argumento nos lleva a preguntarnos qué ocurrió en el caso del aprendizaje de adjetivos. En realidad, el rasgo de la imagen que el niño debía utilizar para elegir el referente era el estampado, siendo el referente específico de la imagen, al igual que el del adjetivo, la propiedad de objeto. En este sentido, no extender la palabra a otro ejemplar que poseía la misma propiedad, denotaría que los niños no decodificaron la propiedad específica de esa imagen.

Si nos adentramos en el campo del aprendizaje de palabras, la comparación del aprendizaje de sustantivos y adjetivos pone de manifiesto que para niños de tres años es más sencillo aprender un sustantivo que un adjetivo mediante libros con imágenes. Los resultados de los estudios presentados son consecuentes con investigaciones que destacan que los niños adquieren más fácilmente palabras que refieren a categorías de objeto que palabras que remiten a propiedades, al menos en inglés, italiano, francés y español (Booth \& Waxman, 2009; Waxman \& Guasti, 2009; Waxman, Senghas \& Benveniste, 1997).

Los resultados muestran que cuando los niños aprendían los adjetivos sin recibir ayuda alguna de la experimentadora realizaban extensiones basados en categorías de objeto y no en propiedades. Es decir, conectaban la palabra pompeado con el objeto que habían visto reflejado en la imagen, pero si este objeto cambiaba no conectaban el adjetivo con el nuevo ejemplar.

El aprendizaje de un adjetivo en este contexto se facilita si los niños cuentan con información descriptiva. En este caso las pistas otorgadas consistían en una descripción que destacaba la característica o propiedad de la imagen, el estampado, con la que debía conectarse la palabra. Recibiendo esta ayuda los participantes extendieron el adjetivo enseñado a otros ejemplares que también presentaban la propiedad pompeado, ejemplares que, incluso, pertenecían a diferentes categorías no solo de nivel básico, sino incluso de nivel supraordenado. La tarea de extender un adjetivo a objetos que no comparten la misma categoría de nivel básico es una tarea difícil no solo para niños, sino también para adultos
(Hall \& Lavin, 2004; Klibanoff \& Waxman, 2000; Medin \& Shoben, 1988).

¿En qué consistía esta pista? Por un lado ponía de relieve las características perceptuales de la imagen que debían conectarse con la palabra pompeado. Podríamos pensar que esta pista específica, facilitaría la conexión asociativa entre ese rasgo perceptual y la palabra escuchada. Esta hipótesis se acercaría a explicaciones asociacionistas para las que la percepción es crucial en la detección del significado de adjetivos (Sandhofer \& Smith, 2004; Smith, 2000; Yee, Jones \& Smith, 2012).

Sin embargo, hay que destacar que el adjetivo enseñado (pompeado) refería a una propiedad visual (estampado) por lo que el corazón del concepto está constituido por las características perceptuales de esa propiedad o estampado. Puesto que la pista ponía de relieve en qué consistía el concepto y cuál sería el patrón de generalización, esta estrategia no desencadenaría sólo asociaciones perceptuales, sino un proceso abstracto de comprensión conceptual. A nuestro modo de ver, esto se refleja en la posibilidad de generalizar la palabra pompeado a un nuevo ejemplar ya que perceptualmente los ejemplares pompeados (por ejemplo, tren, caballo) no compartían muchas similitudes, sólo el atributo de ese estampado. Por lo tanto la conexión se estableció entre la palabra y el concepto $P O M$ $P E A D O$. Esto podría reflejar la intrínseca relación que se establece entre categorización y aprendizaje del léxico.

Cabe destacar que la descripción es altamente útil cuando debemos conectar un adjetivo con una propiedad visual a la que refiere. ¿Pero, qué ocurriría si enseñamos otro tipo de adjetivos? En este sentido, futuras investigaciones podrían explorar las pistas que serían efectivas cuando se trata de enseñar un adjetivo que no refiere a una propiedad visual, por ejemplo una textura.

Por otro lado, la intervención de la experimentadora residía en una guía activa, en una pista socio-pragmática que evidenciaba su intención comunicativa, ya que ponía de relieve qué era para ella un objeto pompeado, en qué consistía la propiedad. Gracias a esta 
pista el niño pudo captar que para el adulto pompeado significaban esos puntos o esos cuadros, y que debía conectar esa parte particular de la imagen, el estampado, con el referente.

En este contexto encontramos que los niños ya a los tres años pudieron utilizar pistas socio-pragmáticas para aprender un adjetivo, mientras que en investigaciones previas (Hall et al., 2010) los participantes las utilizaban recién a los cuatro. Esto podría estar relacionado con las características de la tarea. En la tarea de Hall y colaboradores se estudiaban dos variables, la forma en que el nuevo adjetivo era presentado, pistas de léxico-sintácticas y las pistas socio-pragmáticas. Las primeras mostraron ser más efectivas que las segundas a edades tempranas. En nuestro trabajo los participantes siempre recibían la pistas léxicas, ya que la palabra pompeado era introducida en una oración y ubicada en el lugar de la frase en el cual se ubican los adjetivos, por lo que se variaba sólo la pista sociopragmática.

Sin embargo, en esta ocasión encontramos que las pistas léxico-sintácticas por sí solas no serían suficientes para que a los tres años los niños conecten la palabra pompeado con el concepto POMPEADO. Este tipo de pista debió ser acompañada con una intervención que combinaba pistas socio-pragmáticas (acciones señalamiento) y conceptualreferenciales (denotación del atributo o estampado). Es decir, el niño debía recibir información acerca de la intención comunicativa del adulto y del concepto con el cual debía relacionar la palabra aprendida. Futuros estudios podrían indagar si esta pista ayuda a conectar el adjetivo con la propiedad incluso a edades más tempranas.

Los niños son activos formadores de conceptos pero no pueden acceder a estos por sí solos: requieren conocer cómo las palabras son usadas en su comunidad lingüística. Si consideramos a los significados de las palabras como construcciones sociales, los mismos deben ser aprendidos en contextos sociales de uso. La capacidad de asignar una palabra a su referente se basa en una capacidad para inferir que el hablante pretende nombrar el objeto designado. En este punto, la lectura de la intención es una habilidad fundacional para comprender la función referencial las palabras (Bloom, 2000; Bloom \& Markson, 1998; Nelson, 1996; Tomasello, 2003).

Esta investigación contribuye a pensar al aprendizaje de palabras como un proceso de interrelación entre habilidades cognitivas individuales y el contexto social. Además, pone de relieve las relaciones entre aprendizaje de palabras y desarrollo conceptual. Asimismo, el estudio realizado evidencia que en el aprendizaje de palabras a través de imágenes se interrelacionan dos procesos cognitivos: por un lado la comprensión simbólica de imágenes y por otro lado el aprendizaje del léxico. En este sentido pudimos observar que la enseñanza de distintos tipos de palabras a través de imágenes requiere estrategias específicas $\mathrm{y}$, a su vez, el aprendizaje de distintos tipos de palabras puede impactar en la comprensión referencial de las imágenes.

En términos más generales, los resultados aquí presentados se suman al corpus de investigaciones que destacan el impacto de la instrucción y la enseñanza como factor facilitador en la comprensión simbólica (Peralta \& Salsa, 2011; Peralta, Salsa, Maita \& Mareovich, 2013). También se alinean con estudios que subrayan la importancia de la interacción social en el desarrollo simbólico en general y en el aprendizaje de palabras en particular (Bruner, 1983; Nelson, 2003; Tomasello, 2003). 
TABLA 1

NÚMERO, PORCENTAJE Y COMPARACIÓN DE ELECCIONES EN LA PRUEBA DE GENERALIZACIÓN, POR CONDICIÓN

\begin{tabular}{|l|cccc|c|}
\hline \multirow{2}{*}{$\begin{array}{c}\text { Condición } \\
\text { experimental }\end{array}$} & \multicolumn{4}{|c|}{ Generalización } & Correcto \\
& $n$ & $\%$ & $n$ & $\%$ & $\begin{array}{c}\text { Incorrecto } \\
\text { (Prueba exacta } \\
\text { de Fisher) }\end{array}$ \\
\hline Sin descripción & 3 & 18 & 14 & 82 & .000 \\
Con descripción & 15 & 88 & 2 & 2 & \\
\hline
\end{tabular}

GRÁFICO 1

Porcentaje de ELECCIONES CORRECTAS EN COMPRENSIÓN SIMBÓliCA Y GENERALIZACIÓN, POR CONDICIÓN

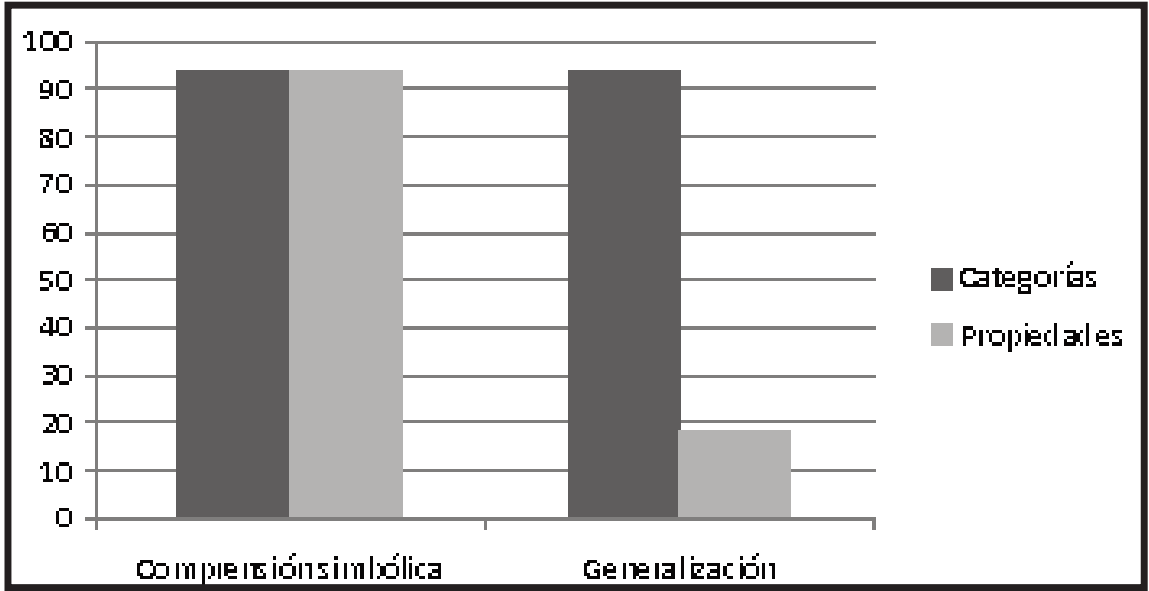




\section{ANEXO}

FIGURA 1

LIBROS UTILIZADOS EN LA FASE DE ENSEÑANZA EN LAS CONDICIONES CATEGORÍA DEL ESTUdIO 1 (IZQUIERDA), PROPIEDAd DEL ESTUdIO 1, SIN DESCRIPCIÓN Y CON DESCRIPCIÓN DEL ESTUdIO 2 (DERECHA)
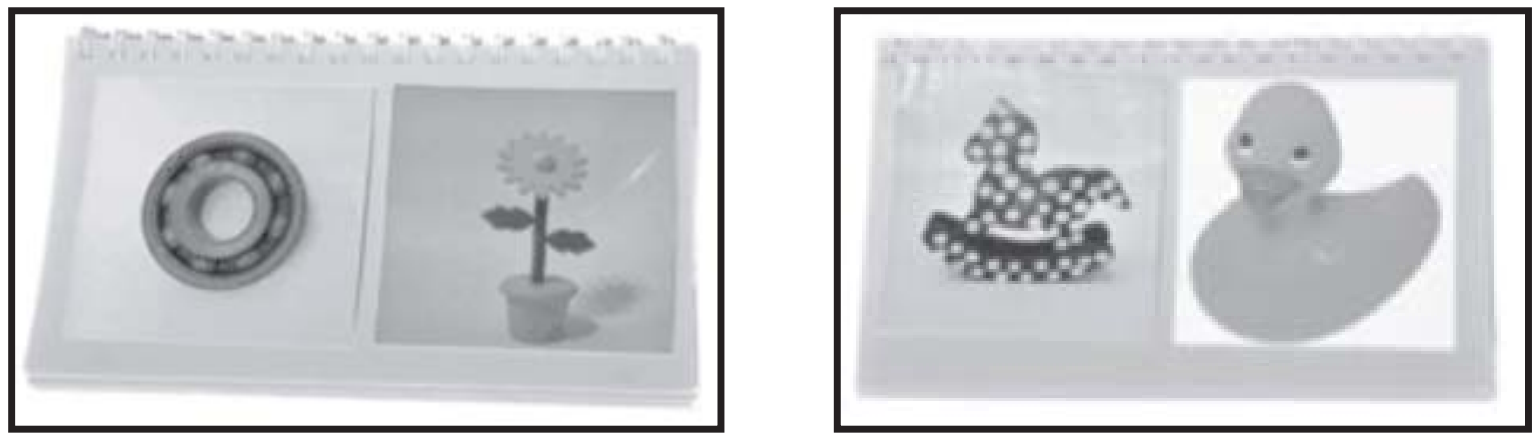

FIGURA 2

OPCIONES PRESENTADAS EN LA PRUEBA DE GENERALIZACIÓN EN LA CONDICIÓN CATEGORÍA DEL ESTUDIO 1(IZQUIERdA), PROPIEdAd del Estudio 1, Sin DESCRIPCIÓN Y CON DESCRIPCIÓN DEL Estudio 2 (DERECHA)
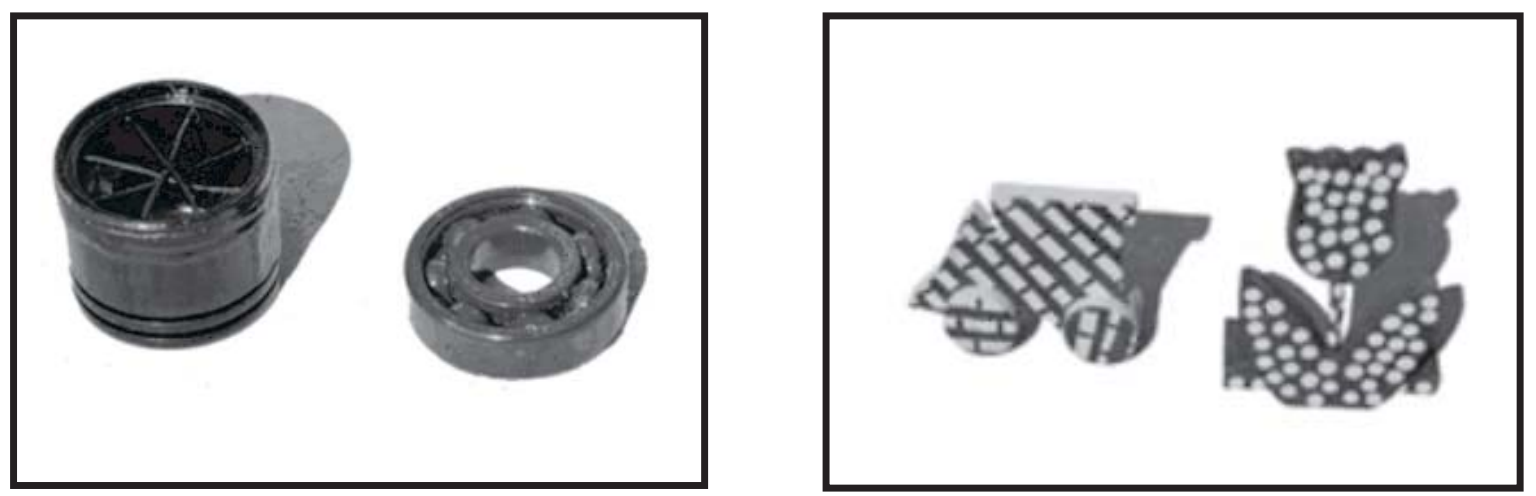


\section{REFERENCIAS BIBLIOGRÁFICAS}

Baldwin, D.A. \& Moses, L.J. (2001). Links between social understanding and early word learning: Challenges to current accounts. Social Development, 10(3), 309-329. http://dx. doi.org/10.1111/1467-9507.00168

Barrera, M. \& Maurer, D. (1981). Recognition of mother's photographed face by the threemonth-old. Child Development, 52(2), 714716.

Bloom, P. (2000). How children learn the meanings of words. Cambridge: MIT Press.

Bloom, P. \& Markson, L. (1998). Intention and analogy in children's naming of pictorial representations. Psychological Science, 9, 200204. http://dx.doi.org/10.1111/1467-9280.00 038

Booth, A. \& Waxman, S. (2009). A horse of a different color: Specifying with precision infants' mappings of novel nouns and adjectives. Child Development, 80(1), 15-22. http://dx.doi.org/ 10.1111/j.1467-8624.2008.01242.x

Booth, A., Waxman, S. \& Huang, Y. (2005). An attentional learning account of the shape bias: Reply to Cimpian and Markman. Developmental Psychology, 42(6). http://dx.doi.org/ 10.1037/0012-1649.42.6.1339

Brent, M.R. \& Cartwright, T.A. (1996). Distributional regularity and phonotactic constraints are useful for segmentation. Cognition, 69(12), 93-125. http://dx.doi.org/10.1016/S00100277(96)007 19-6

Bruner, J. (1983). Child's talk: Learning to use language. Nueva York: Norton.

Callaghan, T. (2008). The origins and development of pictorial symbol functioning. En C. Milbrath \& H. Trautner (Eds.), Children's understanding and production of pictures, drawing, and art (pp. 21-32). Cambridge, MA: Hogrefe \& Huber.
Carey, S. (2009). The origin of concepts. New York: Oxford University Press.

DeBaryshe, B.D. (1993). Joint picture-book reading correlates of early oral language skill. Child Language, 20, 455-461. http://dx.doi. org/10.1017/S0305000900008370

Defior Citoler, S., Fonseca, L., Gottheil, B., Aldrey, A., Jiménez Fernández, G., Pujals, M., Rosa, G. \& Serrano Chica, F.D. (2006). LEE. Test de Lectura y Escritura en Español [Test of Reading and Writing in Spanish]. Buenos Aires: Paidós.

DeLoache, J.S. (1987). Rapid change in the symbolic functioning of very young children. Science, 238, 1556-1557. http://dx.doi.org/10. $1126 /$ scie nc e. 2446392

DeLoache, J. \& Burns, N. (1994). Early understanding of the representational function of pictures. Cognition, 52, 83-110. http://dx.doi.org/ 10.1016/0010-0277(94)90063-9

DeLoache, J.S. \& Peralta, O.A. (1987). Joint picture book interactions of mothers and 1-yearold children. British Journal of Developmental Psychology, 5, 111-123. http://dx.doi.org/10. 1111/j.2044-835X.1987.tb01047.x

DeLoache, J.S., Peralta, O.A. \& Anderson, K.N. (1999). Multiple factors in early symbol use: Instructions, similarity, and age in understanding a symbol-referent relation. Cognitive Development, 14, 299-312. http://dx.doi.org/ 10.1016/s0885-2014(99)00006

DeLoache, J., Pierroutsakos, S., Uttal, D., Rosengren, K. \& Gottlieb, A. (1998). Grasping the nature of pictures. Psychological Science, 9, 205-210. http://dx.doi.org/10.1111/1467-9280. 00039

DeLoache, J., Strauss, M. \& Maynard, J. (1979). Pictures perception in infancy. Infant Behavior and Development, 2, 77-89. http://dx.doi.org/10.1016/S0163-6383(79) 80010-7 
Ganea, P., Allen, M., Butler, L., Carey, S. \& De Loache, J. (2009). Toddlers' referential understanding of pictures. Journal of Experimental Child Psychology, 104, 283-295. http://dx.doi. org/10.1016/j.jecp.2009.05.008

Ganea, P.A., Bloom Pickard, M. \& DeLoache, J. (2008). Transfer between picture books and the real world by very young children. Journal of Cognition and Development, 9, 46-66. http://dx.doi.org/10.1080/152483707018365 92

Gelman, S.A. \& Waxman, S.R. (2009). Taking development seriously: Theories cannot emerge from associations alone. Trends in Cognitive Sciences, 13(8), 332-333. http://dx.doi.org/10. 1016/j.tics.2 009.05.003

Gentner, D. \& Namy, L. (1999). Comparison in the development of categories. Cognitive Development, 14, 487-513. http://dx.doi.org/ 10.1016/S0885-2014(99)00016-7

Geraghty, K., Waxman, S.R. \& Gelman, S. (2014). Learning words from pictures: 15and 17-month-old infants appreciate the referential and symbolic links among words, pictures, and objects. Cognitive Development, 32, 1-11. http://dx.doi.org/10.1016/j.cogdev. 2014.04.003

Gleitman, L.R., Cassidy, K., Nappa, R., Papafragou, A. \& Trueswell, J.C. (2005). Hard words. Language Learning and Development, 1, 23-64. http://dx.doi.org/10.1207/s1547334 111d0101_4

Golinkoff, R.M. \& Hirsh-Pasek, K. (2008). How toddlers begin to learn verbs. Trends in Cognitive Sciences, 12, 397-403. http://dx.doi.org/ 10.1016/j.tics.2008.07.003

Graham, S.A., Booth, A.E. \& Waxman, S.R. (2012). Words are not merely features: Only consistently applied nouns guide 4-year-olds' inferences about object categories. Language Learning and Development, 8(2), 136-145. http://dx.doi.org/10.1080/15475441.2011.59 9304

Hall, D. \& Lavin, T. (2004). The use and misuse of part-of-speech information in word learning: Implication for lexical development. En D. Hall \& S. Waxman (Eds.), Weaving a lexicon (pp. 339-370). Cambridge, MA: MIT Press.

Hall, D. \& Waxman, S. (1993). Assumptions about word meaning: Individuation and basiclevel kinds. Child Development, 64(5), 15501570. http://dx.doi.org/10.1111/j.1467-8624. 1993.tb02970.x

Hall, D., Waxman, S. \& Hurwitz, W. (1993). How 2- and 4-year-old children interpret adjectives and count nouns. Child Development, 64(6), 1651-1664. http://dx.doi.org/10.1111/ j.1467-8624.1993.tb04205.x

Hall, D.G., Williams, S.G. \& Be'Langer, J. (2010). Learning count nouns and adjectives: Understanding the contributions of lexical form class and social-pragmatic cues. Journal of Cognition and Development,11(1), 86-120. http://dx.doi.org/10.1080/15248370903 453592

Harris, P.L., Kavanaugh, R.D. \& Dowson, L. (1997). The depiction of imaginary transformation: Early comprehension of a symbolic function. Cognitive Development, 12, 1-19. http://dx.doi.org/10.1016/S0885-2014(97)9 0028-9

Huebner, C.E. (2000). Community-based support for preschool readiness among children in poverty. Journal of Education for Students Placed at Risk, 5, 291-314. http://dx.doi.org/ 10.1207/S15327671ESPR0503_6

Ittelson, W.H. (1996). Visual perception of markings. Psychonomic Bulletin \& Review, 3, 171187. http://dx.doi.org/10.3758/BF03212416

Karrass, J., VanDeventer, M.C. \& BraungartRieker, J.M. (2003). Predicting shared parentchild book reading in infancy. Journal of 
Family Psychology, 17, 134-146. http://dx. doi.org/10.1037/0893-3200.17.1.134

Keates, J. \& Graham, S.A. (2008). Category markers or attributes. Why do labels guide infants' inductive inferences? Psychological Science, 19(12), 1287-1293. http://dx.doi.org/ 10.1111/j.1467-9280.2008.02237.x

Klibanoff, R. \& Waxman, S. (2000). Basic level object categories support the acquisition of novel adjectives: Evidence from preschool-aged children. Child Development, 71(3), 649-659.

Lonigan, C.J. (1994). Reading to preschoolers exposed: Is the emperor really naked? Developmental Review, 14, 303-323. http://dx.doi. org/10.1006/drev.1994.1011

Mareovich, F. (2013). Función referencial: El aprendizaje de palabras a través de imágenes [Referential function: Learning words through images]. Tesis Doctoral no publicada. Facultad de Psicología, Universidad Nacional de Córdoba. Argentina.

Markman, E. \& Jaswal, V. (2004). Acquiring and using a grammatical form class: Lessons from the proper-count distinction. En D. Hall \& S. Waxman (Eds.), Weaving a lexicon (pp. 371409). Cambridge, MA: MIT Press.

Medin, D. \& Shoben, E. (1988). Context and structure in conceptual combination. Cognitive Psychology, 20(2), 158-190. http://dx.doi.org/ 10.1016/0010-0285(88)90018-7

Meltzoff, A.N. (2002). Elements of a developmental theory of imitation. En A.N. Meltzoff \& W. Prinz (Eds.), The imitative mind: Development, evolution, and brain bases (pp. 19-41). Cambridge: Cambridge University Press.

Mintz, T.H. (2003). Frequent frames as a cue for grammatical categories in child directed speech. Cognition, 90(1), 91-117. http://dx. doi.org/10.1016/S0010-0277(03)00140-9

Mintz, T.H., Newport, E.L. \& Bever, T.G. (2002). The distributional structure of grammatical categories in speech to young children. Cognitive Science, 26, 393-424.

Murphy, C. (1978). Painting in the context of shared activity. Child Development, 49, 371380.

Nelson, K. (1996). Language in cognitive development. The emergence of the mediated mind. Cambridge, MA: Cambridge University Press.

Ninio, A. \& Bruner, J. (1978). The achievement and antecedents of labeling. Journal of Child Language, 5, 1-15. http://dx.doi.org/10.1017/ S0305000900001896

Payne, A.C., Whitehurst, G.J. \& Angell, A.L. (1994). The role of literacy environment in the language development of children from lowincome families. Early Childhood Research Quarterly, 9, 427-440. http://dx.doi.org/10. 1016/0885-2006(94)90018-3

Peralta, O. (1995). Developmental changes and socioeconomic differences in mother-infant picturebook reading. European Journal of Psychology of Education, 10, 261-272. http:// dx.doi.org/10.1007/BF03172920

Peralta, O. \& Salsa, A. (2011). Instrucción y desarrollo en la comprensión temprana de fotografías como objetos simbólicos [Instruction and development in early comprehension of photographs as symbolic objects]. Anales de Psicología, 1, 18-125.

Peralta, O., Salsa, A., Maita, M. \& Mareovich, F. (2013). Scaffolding young children's understanding of symbolic objects. Early Years: An International Research Journal, 33(3), 266274. http://dx.doi.org/10.1080/09575146.201 2.732042

Perner, J. (1991). Understanding the representational mind. London: MIT Press.

Pierroutsakos, S. \& DeLoache, J. (2003). Infants' manual investigation of pictured objects varying in realism. Infancy, 4, 141-156. http://dx. doi.org/10.1207/S15327078IN0401_7 
Preissler, M. \& Carey, S. (2004). Do both pictures and words function as symbols for 18 and 24-month-old children? Journal of Cognition and Development, 2, 185-212. http:// dx.doi.org/10.1207/s15327 647jcd0502_2

Putnam, H. (1973). Meaning and reference. The Journal of Philosophy, 70, 699-711.

Pye, C. (1992). The acquisition of K'iche' Maya. En D.I. Slobin (Comp.), The cross-linguistic study of language acquisition, (Vol. 3, pp. 221-308). Hillsdale, NJ: Erlbaum.

Real Academia Española - RAE (2012). Diccionario de la lengua española [Dictionary of the Spanish language], (avance de la $23^{\mathrm{a}}$ ed.). Recuperado el 30 de marzo de 2013 de http://ww w.rae.es

Rose, A. (1977). Infants' transfer of response between two-dimensional and three-dimensional stimuli. Child Development, 48, 1086-1091.

Sandhofer, C.M. \& Smith L.B. (2004). Perceptual complexity and form class cues in novel word extension tasks: how 4-year-old children interpret adjectives and count nouns. Developmental Science, 7(3), 378-388. http://dx. doi.org/10.1111/j.1467-7687.2004.0035 4.x

Sigel, I. (1978). The development of pictorial comprehension. En B. Randhawa \& W. Coffman (Eds.), Visual learning, thinking and communication (pp. 93-111). Nueva York: Academic Press.

Sloutsky, V.M. (2010). From perceptual categories to concepts: what develops? Cognitive Science, 34, 1244-1286. http://dx.doi.org/10.111 1/j.1551-6709.2010.01129.x

Smiley, S. \& Brown, A. (1979). Conceptual preference for thematic and taxonomic relations: A non-monotonic age trend from preschool to old age. Journal of Experimental Child Psychology, 28, 249-257.

Smith, L. (2000). Learning how to learn words: An associative crane. En R.M. Golinkoff, K.
Hirsh-Pasek, L. Bloom, L.B. Smith, A.L. Woodard, N. Akhtar, M. Tomasello \& G. Hollich (Eds.), Becoming a word learner (pp. 51-80). Nueva York: Oxford University Press.

Smith, L.B. \& Samuelson, L. (2006). An attentional learning account of the shape bias: Reply to Cimpian and Markman (2005) and Booth, Waxman, and Huang (2005). Developmental Psychology, 42, 1339-1343. http:// dx.doi.org/10.1037/0012-1649.42.6.1339

Snow, C. \& Goldfield, B. (1983). Turn the page please: Situation specific language acquisition. Journal of Child Language, 10, 551-570. http://dx.doi.org/10.1017/S03050009000053 65

Spelke, E.S. (2000). Core knowledge. American Psychologist, 55, 1233-1243. http://dx.doi. org/10.1037/0003-066X.55.11.1233

Tardif, T., Fletcher, P., Liang, W., Zhang, Z., Kaciroti, N. \& Marchman, V.A. (2008). Baby's first 10 words. Developmental Psychology, 44, 929-938. http://dx.doi.org/10.1037/00121649.44.4.929

Tare, M., Chiong, C., Ganea, P. \& DeLoache, J.S. (2010). Less is more: How manipulative features affect children's learning from picture books. Journal of Applied Developmental Psychology, 31, 395-400. http://dx.doi.org/ 10.1016/j.appdev.2010.06.005

Taverna, A. \& Peralta, O. (2009). Desarrollo conceptual: Perspectivas actuales en la adquisición temprana de conceptos [Conceptual development: Current approaches to children's early concept acquisition]. Psykhe, 18(1), 49-59. http://dx.doi.org/10.4067/S0718-2228200900 0100005

Tomasello, M. (2003). Constructing a language: A usage-based theory of language acquisition. Cambridge, MA: Harvard University Press.

Tomasello, M. \& Olguin, R. (1993). Twenty-threemonth-old children have a grammatical cate- 
gory of noun. Cognitive Development, 8(4), 451-464. http://dx.doi.org/10.1016/S0885-20 14(05)80004-8

Waxman, S.R. (1999). Specifying the scope of 13-month-olds' expectations for novel words. Cognition, 70, B35-B50. http://dx.doi.org/10. 1016/S0010-0277(99)00017-7

Waxman, S. \& Guasti, M. (2009). Nouns, adjectives, and the acquisition of meaning: New evidence from Italian-acquiring children. Language Learning and Development, 5, 5068. http://dx.doi.org/10.1080/1547544080234 7528

Waxman, S. \& Lidz, J. (2006). Early word learning. En D. Kuhn \& R. Siegler (Eds.), Handbook of child psychology, (6ta. ed.), Vol. 2 (pp. 299-335). Hoboken NJ: Wiley.

Waxman, S. \& Markow, D. (1998). Object properties and object kind: Twenty-one-month-old infants' extension of novel adjectives. Child Development, 69(5), 1313-1329. http://dx.doi. org/10.1111/j.1467-8624.1998.tb06214.x

Waxman, S., Senghas, A. \& Benveniste, S. (1997). A cross-linguistic examination of noun-category bias: Its existence and specificity in
French- and Spanish-speaking preschool-aged children. Cognitive Psychology, 32(3), 183218. http://dx.doi.org/10.1006/cogp.1997.065 0

Wener, H. \& Kaplan, H. (1963). Symbol formation. Nueva York: Wiley.

Woodward, A.L. (2004). Infants' use of action knowledge to get a grasp on words. En D.G. Hall \& S.R. Waxman (Eds.), Weaving a lexicon (pp. 149-172). Cambridge: MIT.

Woodward, A.L. \& Markman, E.M. (1998). Early word learning. En W. Damon, D. Kuhn \& R.S. Siegler (Eds.), Handbook of child psychology, Vol. 2: Cognition, perception, and language (pp. 371-420). New York: Wiley.

Yee, M., Jones, S. \& Smith, L. (2012). Changes in visual object recognition precede the shape bias in early noun learning. Frontiers in Psychology, 3, 533. http://dx.doi.org/10.3389/fp syg.2012.00533

Xu, F., Cote, M. \& Baker, A. (2005). Labeling guides object individuation in 12-month-old infants. Psychological Science, 16, 372-377. http://dx.doi.org/10.1111/j.0956-7976.2005.0 1543.x

\author{
Consejo Nacional de Investigaciones \\ Cientificas y Técnicas (CONICET) \\ Universidad Abierta Interamericana (UAI) \\ Rosario, Provincia de Santa Fe \\ República Argentina
}

Fecha de recepción: 29 de mayo de 2014 Fecha de aceptación: 9 de febrero de 2015 
